OPEN ACCESS

Edited by:

Manuel Martinez,

Polytechnic University of Madrid,

Spain

Reviewed by:

Shin-Lon Ho,

National Chiayi University, Taiwan

Qiang Zhuge,

Nanjing Forestry University, China

*Correspondence:

Minsheng Yang

yangms100@126.com

tThese authors have contributed equally to this work and share first authorship

Specialty section: This article was submitted to Plant Pathogen Interactions, a section of the journal Frontiers in Plant Science

Received: 01 February 2021 Accepted: 03 May 2021

Published: 28 May 2021

Citation:

Ren Y, Zhou X, Dong Y, Zhang J, Wang J and Yang M (2021)

Exogenous Gene Expression and Insect Resistance in Dual Bt Toxin

Populus $\times$ euramericana 'Neva' Transgenic Plants.

Front. Plant Sci. 12:660226 doi: 10.3389/fpls.2021.660226

\section{Exogenous Gene Expression and Insect Resistance in Dual Bt Toxin Populus x euramericana 'Neva' Transgenic Plants}

\author{
Yachao Ren ${ }^{1,2 t}$, Xinglu Zhou ${ }^{1,2 t}$, Yan Dong ${ }^{1,2 t}$, Jun Zhang ${ }^{1,2}$, Jinmao Wang ${ }^{1,2}$ and \\ Minsheng Yang ${ }^{1,2 *}$
}

${ }^{1}$ Forest Department, Forestry College, Hebei Agricultural University, Baoding, China, ${ }^{2}$ Hebei Key Laboratory for Tree Genetic Resources and Forest Protection, Baoding, China

Bacillus thuringiensis $(\mathrm{Bt})$ insecticidal protein genes are important tools in efforts to develop insect resistance in poplar. In this study, the Cry1Ac and Cry3A Bt toxin genes were simultaneously transformed into the poplar variety Populus $\times$ euramericana 'Neva' by Agrobacterium-mediated transformation to explore the exogenous gene expression and insect resistance, and to examine the effects of Bt toxin on the growth and development of Anoplophora glabripennis larvae after feeding on the transgenic plant. Integration and expression of the transgenes were determined by molecular analyses and the insect resistance of transgenic lines was evaluated in feeding experiments. Sixteen transgenic dual Bt toxin genes Populus $\times$ euramericana 'Neva' lines were obtained. The dual Bt toxin genes were expressed at both the transcriptional and translational levels; however, Cry3A protein levels were much higher than those of Cry1Ac. Some of the transgenic lines exhibited high resistance to the first instar larvae of Hyphantria cunea and Micromelalopha troglodyta, and the first and second instar larvae and adults of Plagiodera versicolora. Six transgenic lines inhibited the growth and development of $A$. glabripennis larvae. The differences in the transcriptomes of $A$. glabripennis larvae fed transgenic lines or non-transgenic control by RNA-seq analyses were determined to reveal the mechanism by which Bt toxin regulates the growth and development of longicorn beetle larvae. The expression of genes related to Bt prototoxin activation, digestive enzymes, binding receptors, and detoxification and protective enzymes showed significant changes in A. glabripennis larvae fed Bt toxin, indicating that the larvae responded by regulating the expression of genes related to their growth and development. This study lay a theoretical foundation for developing resistance to $A$. glabripennis in poplar, and provide a foundation for exploring the mechanism of Bt toxin action on Cerambycidae insects.

Keywords: Bt toxin gene, Populus x euramericana 'Neva', insect resistance, Anoplophora glabripennis, RNA-seq, differentially expressed genes 


\section{INTRODUCTION}

Poplar is an economically important tree species, but its large-scale intensive monoculture has been accompanied by increasingly serious problems of disease and insect pests. Poplar pests such as Hyphantria cunea (Lepidoptera), Micromelalopha troglodyta (Lepidoptera), and longicorn beetles can spread widely and cause substantial losses to forestry production. To reduce the losses caused by insect pests and reduce the use of chemical pesticides, the development of new insect-resistant poplar varieties using transgenic technology is particularly important (Ding et al., 2017). Bacillus thuringiensis (Bt) insecticidal crystal protein genes have been transformed into and expressed in many plant species and are the most widely used insect resistance genes (Lu et al., 2010; Mi et al., 2015; Yang et al., 2016). Among them, the CryI and CryIII genes, which confer high specific resistance to Lepidopteran and Coleopteran pests, respectively, are particularly prominent (Wang et al., 2018). Many studies have shown that introduction of a Bt toxin gene into transgenic poplar provides a degree of resistance to target pests (Dong et al., 2014; Xu et al., 2019, 2020); however, the introduction of a single insect resistance gene has limitations, including a narrow insecticidal spectrum and development of tolerance in the pests. To expand the insecticidal spectrum, delay the development of tolerance in pests, and improve the insect resistance of transgenic poplar, recent research has focused on the introduction of bivalent or multivalent insect resistance transgenes (Zhang B. et al., 2011; Zhang et al., 2019; Yang et al., 2016; Zhou et al., 2020).

The application of transgenic Bt toxin research to the development of insect resistance traits has made understanding the mechanism of $\mathrm{Bt}$ toxin action an important area of research. Bt prototoxins reach the midgut of insects and are proteolytically activated by specific proteases, releasing active toxin polypeptides which then bind to specific receptors on epithelial cells. Mechanisms proposed to be responsible for Cry cytotoxic effects include breakdown of the gut epithelium through a pore-formation mechanism in the target membrane or an alternative cell death process involving the adenylyl cyclase/PKA signaling pathway (Zhang et al., 2006; Bravo et al., 2007). These processes involve interactions with specific proteins found in the insect digestive system, including serine proteases (trypsin, chymotrypsin, and so forth) and Bt toxin-binding receptors (cadherins, aminopeptidase $\mathrm{N}$, alkaline phosphatases, ABC transporter, and heat shock protein HSP70) required for pore formation (Xu et al., 2013; Jin et al., 2019). Crybinding receptors have specific affinities for different Cry toxins (Flores-Escobar et al., 2013). In recent years, there have been many reports on the mechanism of CryI toxin (Wang et al., 2019; Shabbir et al., 2019; Fritz et al., 2020; Prabu et al., 2020), while reports on CryIII toxin are relatively fewer in number (Ruiz-Arroyo et al., 2017; Niu et al., 2020). Cry3A toxin has been shown to be toxic to Chrysomelidae insects with high mortality (Wang et al., 2012), but has only an inhibitory effect on Cerambycidae larvae with low mortality (Niu et al., 2011). The inhibitory mechanism of Cry3A toxin remains unclear and there have been few reports on the topic. Studies on transcriptome responses of insects to xenobiotics may contribute to the discovery of novel insect molecular mechanisms for detoxification and tolerance of toxins.

Populus $\times$ euramericana 'Neva', a hybrid of Populus deltoides and Populus nigra L., has good material quality, fast growth, high yield, and is resistant to drought. It is a preferred poplar variety for industrial raw materials and shelter forest in China. To reduce the losses caused by insect pests and to explore the mechanism by which $\mathrm{Bt}$ toxin affects the growth and development of longicorn beetle larvae, two Bt toxin genes, Cry $1 A c$ and Cry $3 A$, were simultaneously transformed into Populus $\times$ euramericana 'Neva' using an Agrobacterium-mediated method to obtain transgenic poplar with high resistance to Lepidopteran and Coleopteran pests. Molecular analyses were performed to identify the integration and expression of exogenous genes in the transgenic lines and assessed their effects on resistance to target insects in indoor feeding experiments. Furthermore, differences in the growth of newly hatched Anoplophora glabripennis larvae fed with transgenic dual Bt toxin Populus $\times$ euramericana 'Neva' or non-transgenic Populus $\times$ euramericana 'Neva' were observed. The transcriptional response of A. glabripennis larvae to $\mathrm{Bt}$ toxin by determining transcriptional differences among larvae in different treatment groups using RNA-seq technology was also explored. These results will lay a theoretical foundation for establishing resistance to A. glabripennis larvae in poplar, and provide a basis for understanding the molecular mechanisms by which Bt toxin regulates longicorn beetle larval growth and development.

\section{MATERIALS AND METHODS}

\section{Plant Materials and Plant Expression Vector}

Tissue culture seedlings of Populus $\times$ euramericana 'Neva' served as the transgenic receptor material. The plant expression vector N5 was constructed by the Forest Genetics and Breeding Laboratory of Hebei Agricultural University and maintained in Agrobacterium tumefaciens GV3101 (with kanamycin and rifampicin resistance). From the left border to the right border, the vector carried the selectable $n p t I I$ marker gene and the dual Bt toxin genes Cry3A (GenBank accession number: M84650.1) and Cry1Ac (GenBank accession number: AF148644.1, with a small amount of base modification). A nuclear matrix attachment region (MAR, GenBank accession number: U67919.1) was present on the outside of the two Bt toxin genes. The T-DNA structure of the vector is shown in Figure 1A.

\section{Insects Used in Experiments}

Hyphantria cunea belongs to Lepidoptera Arctiidae. Eggs were provided by Professor Min Chen of Beijing Forestry University. After hatching, the larvae were used in indoor feeding experiments.

Micromelalopha troglodyta belongs to Lepidoptera Notodontidae. Eggs were collected from Baoding, Hebei 
Province, China. After hatching, the larvae were used in indoor feeding experiments.

Plagiodera versicolora belongs to Coleoptera Chrysomelidae. Eggs and adults were collected from Baoding. After the eggs hatched, the first instar larvae were used in experiments. Nontransgenic poplar leaves were used to feed the larvae from the first to second instar, and the second instar larvae were used in experiments. Captured adults were tested directly.

Anoplophora glabripennis belongs to Coleoptera Cerambycidae. Adult insects were captured in Baoding. After mating, they were placed in insect rearing boxes $(19 \times$ $12.5 \times 7.5 \mathrm{~cm})$, and a small number of fresh leaves and one willow wood segment ( $15 \mathrm{~cm}$ in length and $5-8 \mathrm{~cm}$ in diameter) were placed in the boxes for adults to obtain nutrients and lay eggs. The wood segment was replaced every 3 days. After 1 week, the bark was removed and the eggs were placed in a culture dish ( $9 \mathrm{~cm}$ in diameter) with wet filter paper on the bottom. The eggs were reared in a dark environment at room temperature. After the eggs hatched, the larvae were used in indoor feeding experiments.

\section{Production of Transgenic Dual Bt Toxin Populus x euramericana 'Neva' Lines Agrobacterium-Mediated Leaf Disk Genetic Transformation}

The dual Bt toxin genes were integrated into the genome of Populus $\times$ euramericana 'Neva' using the Agrobacteriummediated leaf disk method. Resistant lines were obtained after kanamycin screening. After propagation, the lines were domesticated and transplanted to the experimental field, watered regularly, and loosened the soil. Transformations were performed according to Liu et al. (2016) with the following modifications: $100 \mu \mathrm{M}$ acetosyringone (AS) was added to the co-culture medium and the concentration of kanamycin in rooting medium was increased to $50 \mathrm{mg} \cdot \mathrm{L}^{-1}$ to reduce the occurrence of false positives during screening.

\section{Polymerase Chain Reaction (PCR) Detection of Exogenous Genes}

In early June, the leaves of kanamycin-resistant lines and non-transgenic Populus $\times$ euramericana 'Neva' were collected from the experimental field and genomic DNA was extracted by an improved cetyltrimethylammonium bromide (CTAB) method (Wang and Fang, 2002). PCR detection was performed using specific primers for the nptII, Cry1Ac, and Cry $3 A$ genes (primers 103\#, 105\#, and 106\#; Supplementary Table 1). The plasmid N5 was used as a positive control and nontransgenic Populus $\times$ euramericana 'Neva' plants served as a negative control. PCR reactions were performed according to Liu et al. (2016) with a slight modification: the annealing temperature for the $\operatorname{Cry} 3 \mathrm{~A}$ gene was $52^{\circ} \mathrm{C}$. The theoretical sizes of the amplified fragments of the nptII, CrylAc, and $C r y 3 A$ genes were 473, 546, and 667 bp, respectively. PCR product sizes were observed by $1 \%$ TAE agarose gel electrophoresis.

\section{Detection of Exogenous Gene Expression in Transgenic Lines}

Fluorescence Quantitative PCR (FQ-PCR) Detection

In early July, three biological replicate samples of fully expanded young leaves from all transgenic lines and nontransgenic Populus $\times$ euramericana 'Neva' $(\mathrm{CK})$ were collected from the field, frozen in liquid nitrogen, and stored at $-80^{\circ} \mathrm{C}$. Total RNA was extracted and reverse transcribed to produced first-strand cDNA using RNA extraction and reverse transcription kits (SENO, Zhangjiakou, Hebei, China) according to the manufacturer's instructions. The FQ-PCR primers 176\# and 1291\# (amplified target fragments were 176 and 203 bp, respectively; Supplementary Table 1) were designed according to the full Cry1Ac and Cry3A nucleotide sequences using Primer Premier 6.0 (Premier Biosoft, Canada) software. Absolute FQ-PCR was performed using AceQ qPCR SYBR Green Master Mix (Vazyme Biotech Co., Ltd., Nanjing, China) and an Agilent Technologies Stratagene Mx3005P Real-Time PCR instrument (Agilent, United States) to determine the transcriptional expression levels of the exogenous genes. FQ-PCR was performed according to Liu et al. (2016).

\section{Bt Toxin Detection in Transgenic Lines}

The presence of $\mathrm{Bt}$ toxin proteins in the same samples described above in section of "Fluorescence quantitative PCR (FQ-PCR) detection" was detected using Bt-CrylAb/1Ac and Bt-Cry3A ELISA kits (Agdia, United States) according to the manufacturer's instructions. The positive controls used were included in the kits, and the negative control was CK. The results were determined using a Bio-RAD Model 550 microplate reader (Bio-Rad). The toxin content was calculated as the amount of toxin in each gram fresh weight of leaves (ng.g ${ }^{-1} \mathrm{FW}$ ).

\section{Insect Resistance of Transgenic Lines Lepidopteran Target Insect Feeding Experiment}

From late July to early August, the top third or fourth fresh leaves of transgenic and CK lines were collected to conduct indoor feeding experiments on the first instar (L1) larvae of $H$. cunea and $M$. troglodyta. The petioles were inserted into humid flower mud $(2 \times 2 \times 2 \mathrm{~cm})$ to maintain freshness of the leaves. Thirty L1 larvae were placed on each leaf with a brush and placed in a breathable feeding bottle $(6.5 \mathrm{~cm}$ in diameter at the bottom and $8 \mathrm{~cm}$ in height). The leaves and flower mud were replaced daily, and larval mortality was recorded. Each treatment was repeated three times. After the number of surviving larvae in the treatment and control groups stabilized ( $H$. cunea were fed for 14 days and $M$. troglodyta were fed for 10 days), the experiment was terminated. The data were recorded and the relevant indexes were calculated according to the following formulas.

$$
\text { Mortality rate }=\frac{\text { number of deaths }}{\text { total number of tested insects }} \times 100 \%
$$




$$
\begin{aligned}
& \text { Corrected mortality rate }= \\
& \qquad \begin{array}{l}
\frac{(\text { mortality rate of transgenic lines - mortality rate of control })}{(1-\text { mortality rate of control })} \\
\quad \times 100 \%
\end{array}
\end{aligned}
$$

\section{Coleopteran Target Insect Feeding Experiment}

In mid-August, the top third or fourth fresh leaves of transgenic and CK lines were collected to conduct indoor feeding experiments on the first instar (L1) larvae, second instar (L2) larvae, and adults of $P$. versicolora, with 30,20 , and 10 insects, respectively, included in each replicate. Other feeding methods were the same as above. After feeding for 3, 20, and $22 \mathrm{~d}$, respectively, the experiments were terminated. The data were recorded and the relevant indexes were calculated using the same methods as above.

In late August, six transgenic lines $(1,7,9,11,14$, and 15) and CK were selected to feed newly hatched $A$. glabripennis larvae. The weights of the newly hatched larvae were recorded using a milligram balance, and the larvae were transferred to a feeding box $(25 \mathrm{~mL})$ with soft tweezers. The xylem and leaves of tree segments were mixed and crushed to use as feed. The feeding boxes were numbered, each box contained one larva, and each treatment had ten replicates. All feeding boxes were placed in a dark environment at room temperature. The feed was replaced every other day, the mortality was recorded, and the surviving larvae were weighed. After feeding for 35 days, differences between treatments were significant and the experiment was terminated. Each insect was weighed and stored in a centrifuge tube at $-80^{\circ} \mathrm{C}$ for use in subsequent enzyme activity determination and RNA-seq analyses. The mortality, weight increment, and rate of A. glabripennis larval growth inhibition were calculated according to formulas (1), (3), and (4).

Increment $=$ measured value at the end of the experiment

- measured value at the beginning of the experiment

Growth inhibition rate $=$

$\frac{\text { (average increment of control group - average increment of treated group) }}{\text { average increment of controlgroup }}$ $\times 100 \%$

\section{Determination of Cellulase Activity in the Midgut of A. glabripennis Larvae}

Cellulase is a complex enzyme, composed mainly of exo$1,4-\beta$-D-glucanase (C1 enzyme), endo-1,4- $\beta$-D-glucanase ( $\mathrm{Cx}$ enzyme), and $\beta$-1,4-glucosidase ( $\beta$-glucosidase). In this study, the activities of $\mathrm{Cx}$ enzyme and $\beta$-glucosidase in the $A$. glabripennis midgut were determined. The A. glabripennis larvae stored at $-80^{\circ} \mathrm{C}$ were thawed at $4^{\circ} \mathrm{C}$ for $4 \mathrm{~h}$, and each treatment was repeated three times. The intestinal tract was dissected on ice, homogenized on ice in $2 \mathrm{~mL}$ cold acetic acid-sodium acetate buffer solution $\left(\mathrm{pH} 5.2,0.1 \mathrm{~mol} \cdot \mathrm{L}^{-1}\right)$, and centrifuged at $12,000 \mathrm{r} \cdot \mathrm{min}^{-1}$ for $20 \mathrm{~min}$ at $4^{\circ} \mathrm{C}$. The supernatant containing the isolated enzymes was stored at $-30^{\circ} \mathrm{C}$.

The cellulase components were determined by the 1, 3dinitrosalicylic acid (DNS) method (Shi et al., 2011), and a glucose standard curve was drawn according to Sinegani and Emtiazi (2006). Cx enzyme activity was determined using $1 \%$ carboxymethyl cellulose (CMC) as the substrate, while $\beta$ glucosidase activity was determined with $1 \%$ salicylic acid as substrate according to the method of Li et al. (2011). Each treatment was repeated three times. Enzyme activity $(\mu \mathrm{mol}$ glucose $\cdot \mathrm{g}^{-1} \mathrm{FW} \cdot \mathrm{h}^{-1}$ ) was calculated according to the amount of reducing sugar (glucose) produced by enzymatic reaction per unit body weight (FW) per unit time (h).

\section{Transcriptome Sequencing RNA Extraction, cDNA Library Preparation, and RNA-Seq Analyses}

Based on the results of the feeding experiments and the cellulase activities of A. glabripennis larvae, the larvae that were fed with transgenic lines 7 and 15 and with CK were selected for RNA-seq analyses, and named treatment groups A, B, and $\mathrm{CK}$, respectively. Larvae with the same growth vigor were selected for each treatment. Each treatment was repeated three times with one larva per replicate. Total RNA was extracted by the TRIzol method. The quality and concentration of RNA were determined using an Agilent 2100 Bioanalyzer (Agilent Technologies, Palo Alto, CA, United States) and a NanoDrop spectrophotometer (Thermo Fisher Scientific Inc.). Three cDNA libraries were constructed and after quality inspection, were sequenced using an Illumina HiSeq 2000 high-throughput sequencing platform.

\section{Sequencing Quality Evaluation, Sequence Alignment, Screening, and Functional Annotation of Differentially Expressed Genes (DEGs)}

Clean data were obtained after quality evaluation and preprocessing of sequencing data using FastQC (ver. 0.10.1) and Cutadapt (ver. 1.9.1) software, and compared against the $A$. glabripennis reference genome $e^{1}$ using Hisat2 (ver. 2.0.1) software. Gene annotation was performed according to the A. glabripennis reference genome annotation document ${ }^{2}$. Gene expression levels were calculated as fragments per kilobase of exon model per million reads mapped (FPKM) with Htseq (ver. 0.6.1) software, and the DEGs among samples were analyzed using DESeq2 (ver. 1.6.3) software. The formula used to calculate the fold-change (FC) between the two groups was $\log _{2} \mathrm{FC}=\log _{2}$ (treatment group/control group). A gene was designated as a DEG when $\left|\log _{2} \mathrm{FC}\right|>1$ and $P$-value $<0.05$. A two-sample differential expression analysis method was applied to identify the number of DEGs, and the numbers of upregulated and downregulated genes were calculated according to the $\log _{2} \mathrm{FC}$ value. K-means hierarchical clustering software was used for cluster analyses of the expression patterns of all DEGs. Gene Ontology (GO)

\footnotetext{
${ }^{1}$ https://www.ncbi.nlm.nih.gov/genome/?term=Anoplophora+glabripennis ${ }^{2} \mathrm{ftp}$ ://ftp.ncbi.nlm.nih.gov/genomes/all/GCF/000/390/285/GCF_000390285.2_ Agla_2.0/GCF_000390285.2_Agla_2.0_genomic.gff.gz
} 
and Kyoto Encyclopedia of Genes and Genomes (KEGG) databases were used for functional annotation, classification of DEGs, and enrichment analyses of metabolic pathways. Heatmaps were generated using the among-samples normalized FPKM values as an indicator of gene expression values with the Pheatmap R package.

\section{Verification of DEGs With Real-Time Quantitative Reverse Transcription PCR (RT-qPCR)}

Seven genes were randomly selected from the RNA-seq results. The glyceraldehyde-3-phosphate dehydrogenase (GAPDH, GenBank accession number: KU521365.1) gene served as the reference gene. The reliability of RNA-seq results was verified by RT-qPCR. Primers were designed using Primer Premier 6.0 software. The primer information is presented in Supplementary Table 1. The HiScript III RT SuperMix for qPCR (+gDNA wiper) reverse transcription kit (Vazyme Biotech Co., Ltd., Nanjing, China) was used for reverse transcription of the samples after sequencing. RT-qPCR analyses were conducted using AugeGreen qPCR Master Mix (US Everbright Inc., Suzhou, China) and the Agilent Technologies Stratagene Mx3005P Real-Time PCR instrument (Palo Alto, CA, United States) according to Ren et al. (2020). Each sample was repeated three times. Relative expression was calculated by the $2^{-\Delta \Delta \mathrm{CT}}$ method.

\section{Statistical Analyses}

Excel 2016 (Microsoft, Inc., Redmond, WA, United States) and Data Processing System (DPS) 7.05 (China) software were used to consolidate and analyze the data. The data were analyzed for each treatment using one-way analysis of variance (ANOVA), and significance was tested using Duncan's multiple range test $(P<0.05)$. Excel 2016 software was used to create tables and OriginPro 2018 (OriginLab Corporation, Northampton, MA, United States) software was used to create drawings.

\section{RESULTS}

\section{Production of Dual Bt Toxin Populus x euramericana 'Neva' Transgenic Lines and Confirmation of Exogenous Genes by PCR}

Agrobacterium tumefaciens harboring the transformation construct containing the Cry $1 A c$ and Cry $3 A$ genes was inoculated into incisions in leaf disks resulting in production of green buds in some of the incisions. Kanamycin-resistant buds were transferred to rooting medium containing kanamycin. After 5-7 days, most of the resistant buds grew roots. The shoots of the rooting plants were transferred to differentiation selection medium to propagate. Then kanamycin-resistant shoots were induced to root, the rooted plantlets were transplanted into small flowerpots, and the plants were subsequently transplanted to the experimental field. In total, 16 transgenic dual Bt toxin Populus $\times$ euramericana 'Neva' kanamycin-resistant rooted lines with were obtained. Presence of the Cry1Ac, Cry3A, and
nptII, transgenes in the lines was confirmed by PCR followed by gel electrophoresis (Figure 1B). Bands corresponding to the three exogenous genes were amplified in all transgenic lines and the positive control, but not detected in the negative control, indicating that the exogenous genes had integrated into the Populus $\times$ euramericana 'Neva' genome.

\section{Transgene Expression Levels in Transgenic Lines}

The Bt toxin transgene transcript abundances and protein contents of transgenic lines are shown in Figure 2. The Cry1Ac transcript abundances ranged from $1.15 \mathrm{E}+03$ to $9.50 \mathrm{E}+05$, with the highest expression in line 7 and the lowest expression in line 13. The Cry $3 A$ transcript abundances ranged from $4.06 \mathrm{E}+04$ to $1.21 \mathrm{E}+06$, with the highest expression in line 7 and the lowest expression in line 10. The fluorescence signals corresponding to the two genes were not detected in CK. The CrylAc and Cry3A transcript abundances differed among the lines, with significant differences found between some lines. The coefficients of variation of Cry1Ac and Cry $3 A$ transcript abundance were 1.014 and 0.865 , respectively. The correlation coefficient of the two genes was $0.634(P<0.01)$ (Supplementary Table 2 ).

Bt toxin protein expression was detected in all transgenic lines, but there were differences among the lines, some of which were significant. CrylAc toxin content ranged from $0.591 \pm 0.182$ to $3.453 \pm 0.336 \mathrm{ng} \cdot \mathrm{g}^{-1} \mathrm{FW}$, with the highest content in line 14 and the lowest content in line 2, while Cry3A toxin content ranged from $373.485 \pm 77.232$ to $12,308.543 \pm 1,030.893 \mathrm{ng} \cdot \mathrm{g}^{-1}$ FW, with the highest content in line 6 and the lowest content in line 1. The Cry3A toxin content was much higher than that of Cry1Ac. The coefficients of variation of Cry1Ac and Cry3A toxin content were 0.430 and 0.906 , respectively, and the correlation coefficient was $0.554(P<0.05)$ (Supplementary Table 2) indicating a significant correlation. The correlation coefficients between the transcript abundances of Cry $1 A c$ and $C r y 3 A$ and the corresponding toxin proteins were $0.644(P<0.05)$ and 0.535 $(P<0.05)$, respectively (Supplementary Table 2$)$, indicating a significant correlation between the transcript abundance and the toxin protein content.

\section{Resistance of Transgenic Lines to Target Insects Resistance of Transgenic Lines to Lepidopteran Pests}

The lethal effects of the transgenic lines on the L1 larvae of $H$. cunea and M. troglodyta are shown in Figure 3 and photographs of feeding experiments are presented in Supplementary Figure 1. Insect resistance levels differed among the transgenic lines. The corrected mortality rates of $H$. cunea L1 larvae fed transgenic lines $1,6,7,9,11,14$, and 15 reached $100 \%$, while those of larvae fed lines 4,5 , and 8 were greater than $93 \%$. The other lines caused lower mortality rates, with line 12 causing the lowest mortality (12.50\%). The corrected mortality rates of $M$. troglodyta L1 larvae fed transgenic lines 1, 6, 7, 11, and 14 reached 100\%, and those of larvae fed lines 5, 8, 9, 15, and 16 were greater than $94 \%$. The mortality rates caused by other lines were lower, 


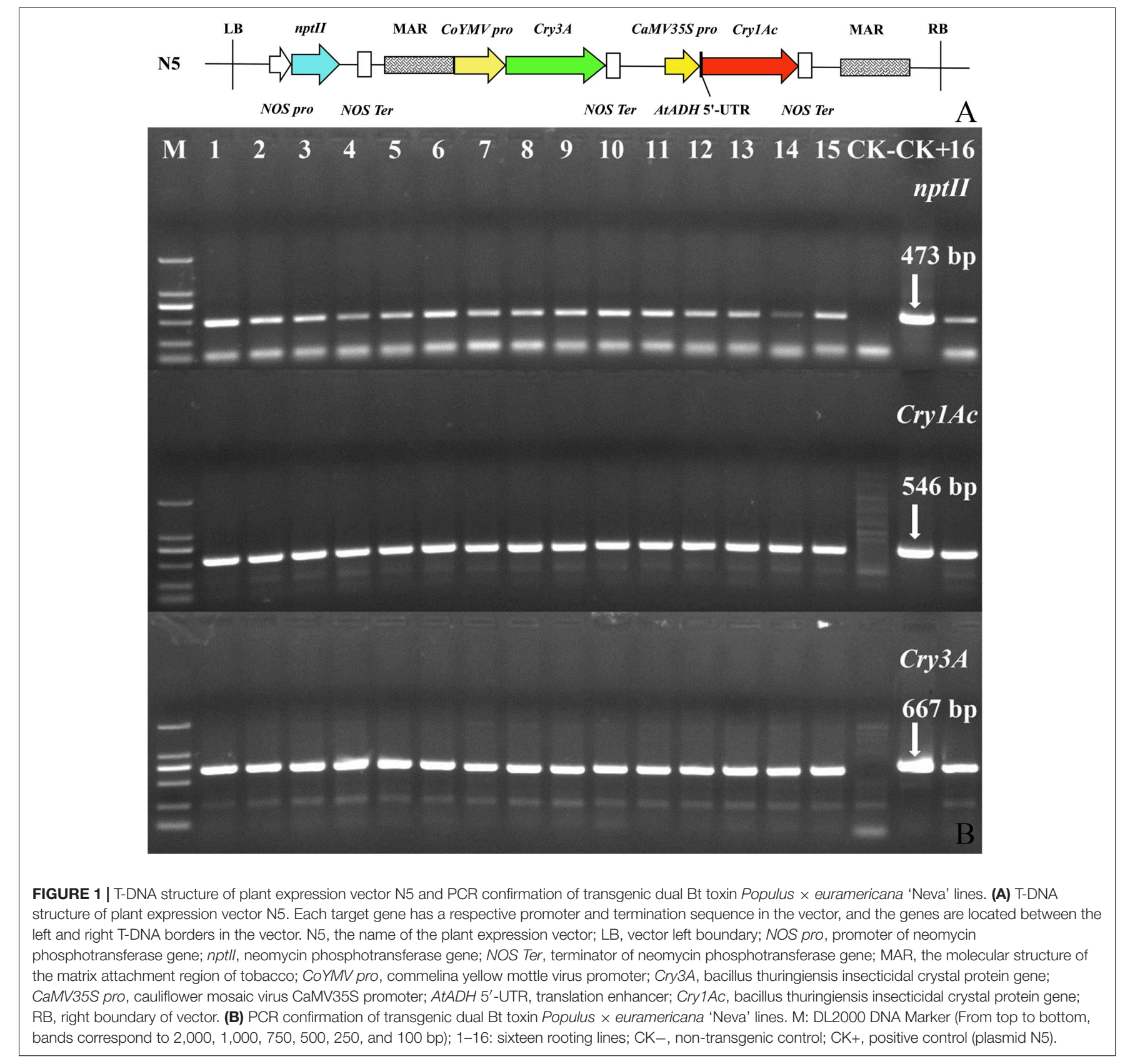

with line 4 causing the lowest mortality (16.21\%). The transgenic lines exhibited resistance to the L1 larvae of $H$. cunea and $M$. troglodyta, with lines $1,6,7,11$, and 14 exhibiting the highest levels of resistance. The correlation coefficients between CrylAc toxin content and the corrected mortality rates of $H$. cunea and $M$. troglodyta L1 larvae were $0.668(P<0.01)$ and 0.419 $(P<0.05)$, respectively (Supplementary Table 2$)$, indicating extremely significant and significant correlations, respectively.

\section{Resistance of Transgenic Lines to $P$. versicolora (Coleoptera)}

The lethal effects of the transgenic lines on the L1 and L2 larvae and adults of $P$. versicolora are shown in Figure 3, and photographs of feeding experiments are shown in Supplementary Figure $\mathbf{1}$. The levels of insect resistance differed among the transgenic lines, with some differences being significant. The corrected mortality rates of $P$. versicolora L1 larvae after feeding reached $100 \%$ for all of the transgenic lines. The corrected mortality rates of $P$. versicolora L2 larvae reached $100 \%$ for most of the transgenic lines with only line 2 causing a slightly lower mortality rate of $96.21 \%$. The corrected mortality rates of adults reached $100 \%$ for most lines, and the corrected mortality rates of lines $1,2,3,4,10,12$, and 13 ranged from $4.17 \%$ to $66.0 \%$, with lines 1 and 3 exhibiting the least resistance. On the whole, lines 5, 6, 7, 8, 9, 11, 14, 15 , and 16 exhibited high resistance to L1 and L2 larvae and 

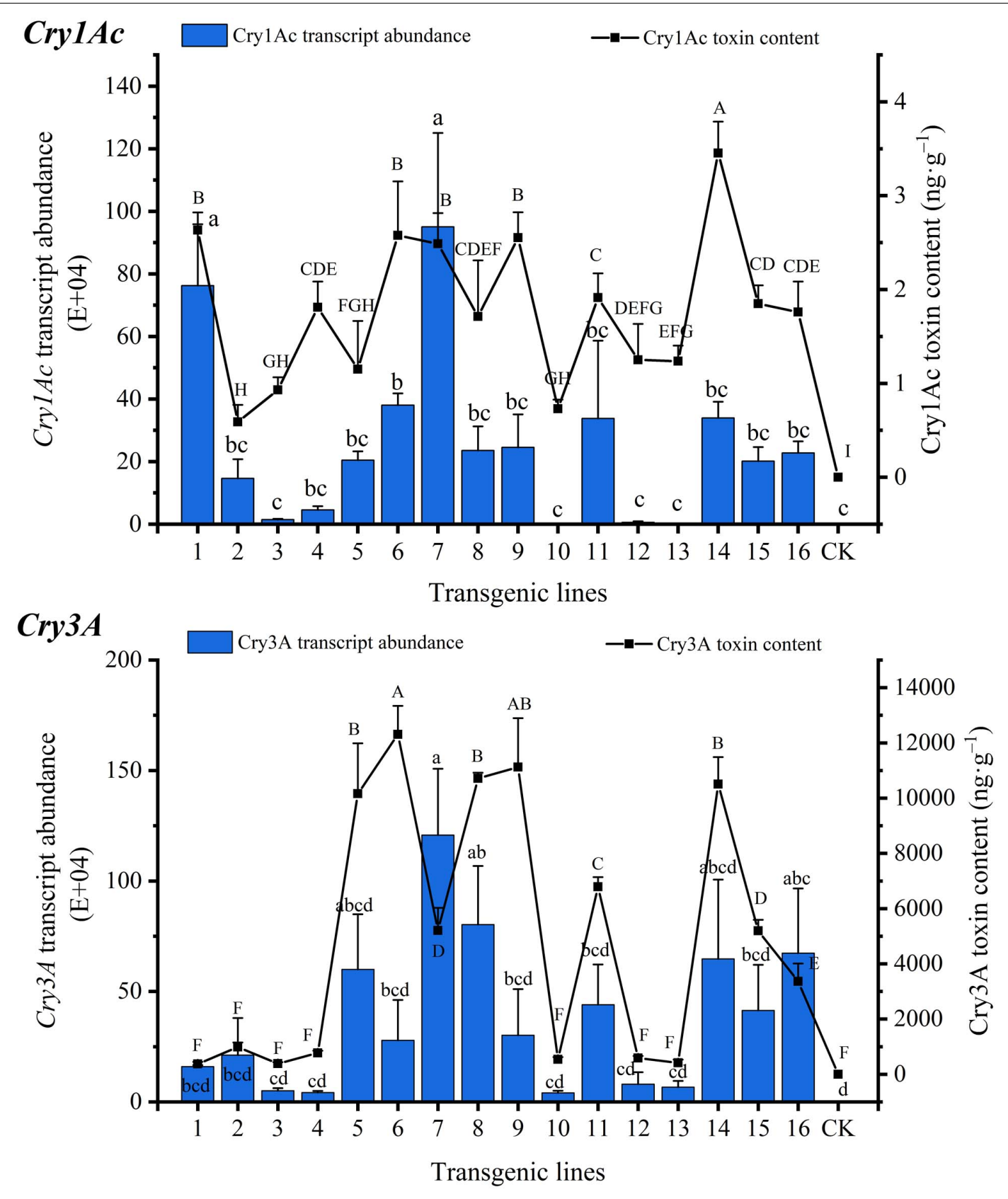

FIGURE 2 | Transgene transcript abundance and Bt toxin protein content in transgenic lines. Lowercase letters indicate multiple comparisons of the transcript abundance of the transgenic lines $(P<0.05)$, and capital letters indicate multiple comparisons of the Bt toxin protein contents of the transgenic lines $(P<0.05)$.

adults of $P$. versicolora. The correlation coefficients between Cry3A toxin content and corrected mortality rates of $\mathrm{L} 1$ and L2 larvae and adults were 0 and $0.363(P<0.05)$, and 0.810 $(P<0.01)$, respectively (Supplementary Table 2$)$. The latter two correlation coefficients were significant and extremely significant, respectively.

Based on the observed lethal effects of the transgenic lines on H. cunea, M. troglodyta, and P. versicolora, lines 5, 6, 7, 8, 9, 11, 14 , and 15 exhibited strong insect resistance, while line 1 showed poor resistance against $P$. versicolora adults.
Inhibitory Effects of Transgenic Lines on A. glabripennis Larval Growth and Development

During feeding on the transgenic lines by A. glabripennis larvae, only one larva died on lines $7,9,11$, and 15 (10\% mortality). The variation in the average weights of A. glabripennis larvae is shown in Figure 4A. At the beginning of feeding, changes in larval weight were essentially the same among the larvae. After 9 days of feeding, differences began to appear and gradually became obvious. After 23 days, the weights of the larvae fed the control increased rapidly, but those of the larvae fed the 


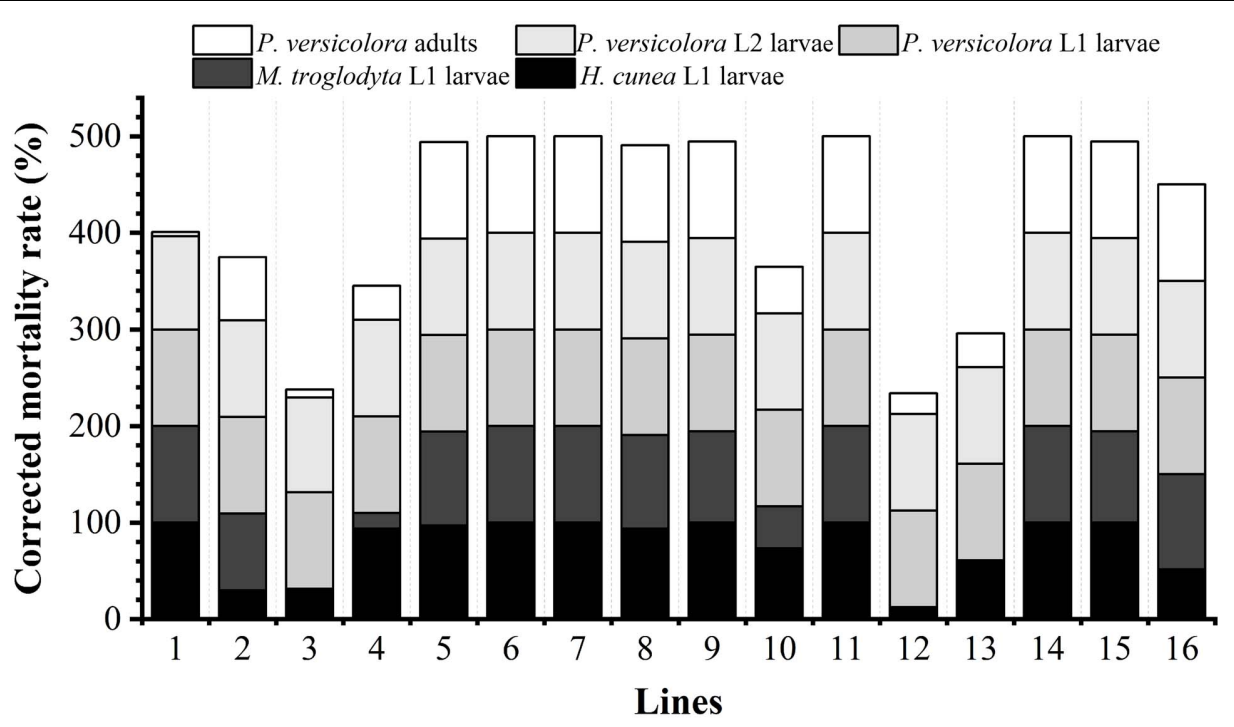

FIGURE 3 | Resistance of transgenic lines to target insects. M. troglodyta L1 larvae were not fed lines 3, 12 , or 13.

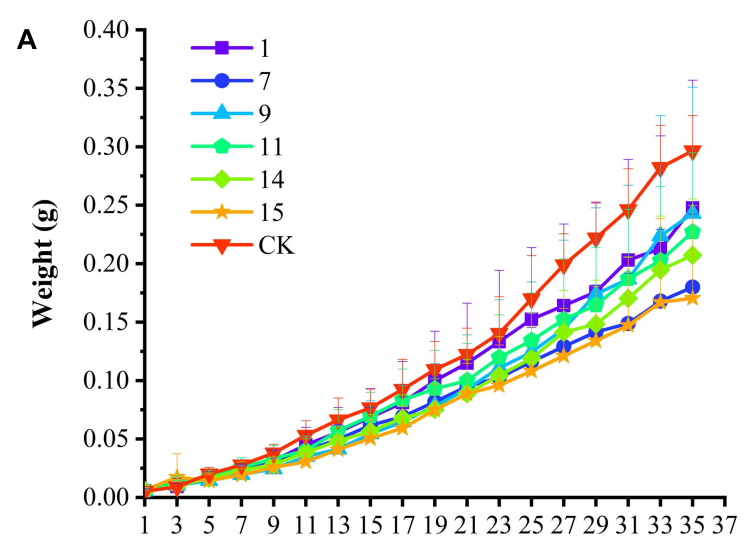

Feeding days (d)

C

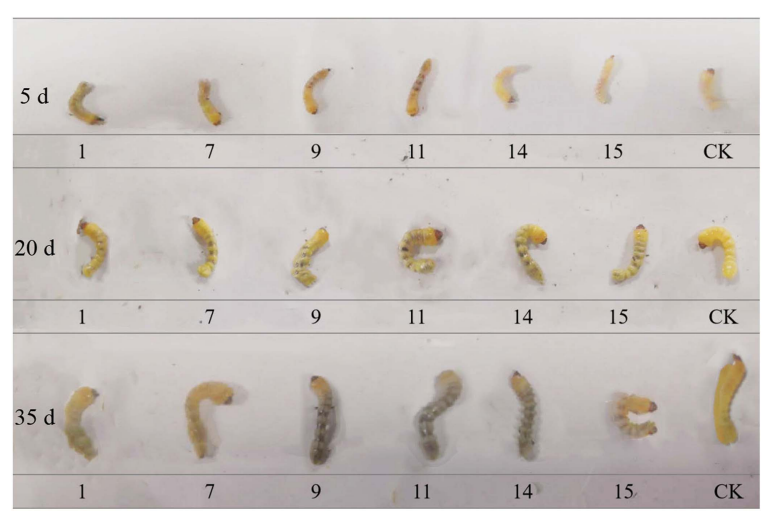

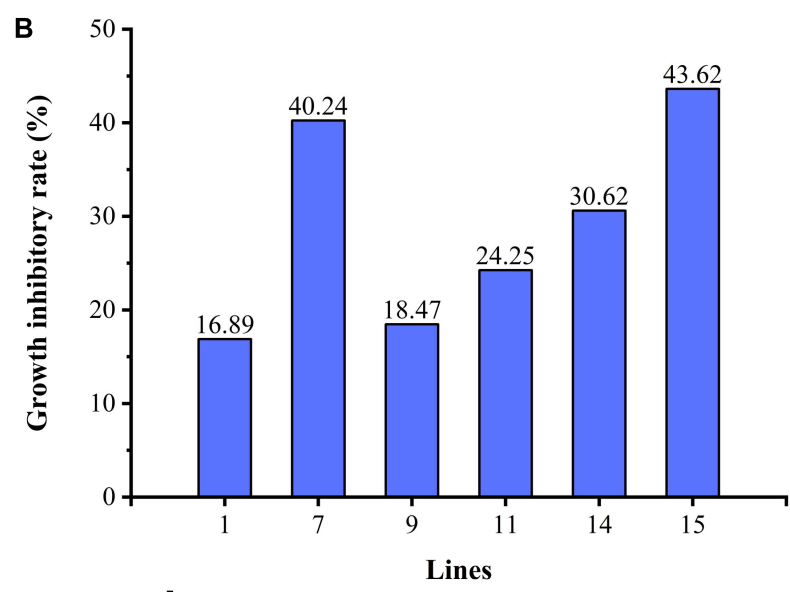

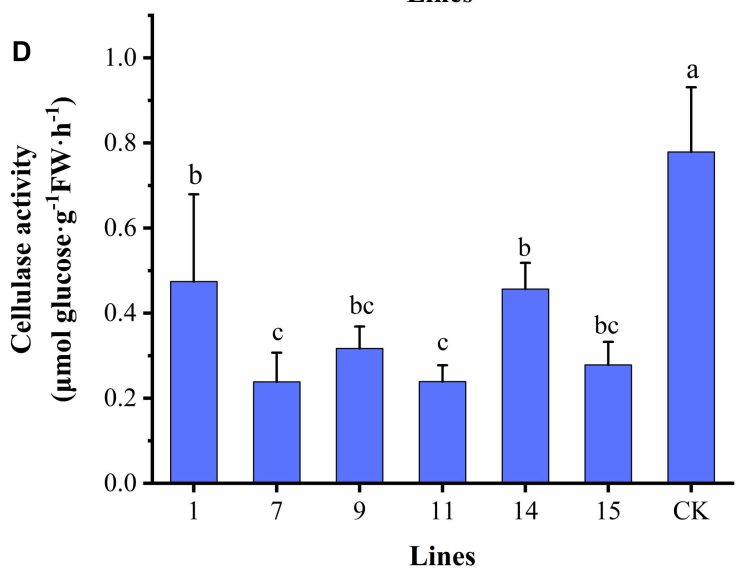

FIGURE 4 | Growth indexes of $A$. glabripennis larvae. (A) Variation in average body weights of $A$. glabripennis larvae fed different lines. (B) Rates of growth inhibition in A. glabripennis larvae fed different transgenic lines. (C) Comparison of the larvae fed the different lines. (D) Cellulase activities in the midguts of larvae fed different transgenic lines. 
transgenic lines increased relatively slowly. On day 35, the weights of the larvae showed hierarchical differentiation. Larvae fed $\mathrm{CK}$ had the highest weights, followed by those fed lines $1,9,11$, and 14 , and the larvae with the lowest weights were those fed lines 7 and 15. There were differences in the weight increment of $A$. glabripennis larvae fed the various transgenic lines. The growth inhibition rates of $A$. glabripennis larvae fed transgenic lines are shown in Figure 4B. The growth inhibition rate reached more than $40 \%$ for the larvae fed lines 7 and 15 , indicating strong inhibition, while the growth inhibition rates of the other four lines ranged from $16.89 \%$ to $30.62 \%$. A comparison of larval growth and development is shown in Figure 4C. The larvae fed transgenic lines were slightly blackened, while those fed CK were white. On day 35, the larvae fed lines 7 and 15 were significantly smaller than those fed CK, and were curled and exhibited lower activity, indicating that these two transgenic lines had strong inhibitory effects on the growth and development of A. glabripennis larvae, while there was little difference between the other lines and CK. Cellulase activity levels in the larval midgut are shown in Figure 4D. The cellulase activities were significantly lower in the midguts of larvae fed the transgenic lines than in those fed CK, and the cellulase activities in the larvae fed lines 7,11 , and 15 were less than $36 \%$ of those fed CK. There was a significant correlation coefficient of $0.789(P<0.05)$ between cellulase activity and body weight (Supplementary Table 2). These results suggest that Bt toxin can indirectly affect the growth and development of A. glabripennis larvae by inhibiting cellulase activity in the midgut.

\section{Transcriptional Response of A. glabripennis Larvae to $\mathrm{Bt}$ Toxin}

After quality control to remove sequencing junctions, contamination, and low-quality data, $59.5 \mathrm{~Gb}$ of clean data were obtained from RNA-seq analyses (Supplementary Table 3). The mapped rate of each sample was $>81 \%$, the GC content ranged from $44.40 \%$ to $47.17 \%$, and the Q30 was $>93 \%$ (Supplementary Table 3), indicating that the sequencing data were suitable for further analyses.

\section{Identification of DEGs and Hierarchical Cluster Analyses}

To explore the mechanism of $\mathrm{Bt}$ toxin inhibition of $A$. glabripennis larval growth and development, DEGs were identified meeting criteria of $P<0.05$ and $\left|\log _{2} \mathrm{FC}\right|>1$. The statistical results are shown in Figure 5A. Comparison of treatment group A with control group CK (A vs. CK) identified 395 DEGs of which 181 genes were downregulated and 214 genes were upregulated. There were also 395 DEGs identified in the comparison of treatment group B compared with CK (B vs. CK), including 201 downregulated genes and 194 upregulated genes. There were 88 DEGs shared between the two comparisons, of which 86 DEGs had consistent expression patterns, while those of 2 DEGs differed between the comparisons (Supplementary Table 4).

K-means hierarchical clustering analyses were performed on all of the DEGs in the two comparative analysis groups.
The results clustered into four expression patterns (Figure 5B). Subcluster_1, subcluster_2, subcluster_3, and subcluster_4 contained 269, 60, 29, and 344 DEGs, respectively. Among them, subcluster_1, subcluster_2, and subcluster_3 showed gene expression levels that were higher in treatment groups $A$ and $B$ than in $\mathrm{CK}$, and the changes in gene expression were more significant in treatment group B. By contrast, subcluster_4 showed gene expression levels that were lower in treatment groups $\mathrm{A}$ and $\mathrm{B}$ than in $\mathrm{CK}$, and the gene expression changes were more significant in treatment group A.

\section{GO Functional Enrichment and KEGG Annotation of Metabolic Pathways}

Functional annotation of DEGs was performed according to the GO database (Figure 5C). DEGs of A vs. CK belonged to 14 subclasses in molecular functions and biological processes $(Q$-value $<0.05)$ and were mainly enriched in structural constituent of cuticle, hydrolase activity, response to oxidative stress, oxidoreductase activity, catalase activity, and inositol biosynthesis process. DEGs of B vs. CK belonged to 24 subclasses in biological processes, cellular components, and molecular functions, which were mainly enriched in monooxygenase activity, heme binding, oxidoreductase activity, acireductone synthase activity, cadherin binding, and inositol biosynthetic process. The types of GO terms enriched for among the DEGs differed between treatment groups $\mathrm{A}$ and $\mathrm{B}$.

Functional annotation and pathway analyses were performed according to the KEGG database. Five DEGs of A vs. CK were enriched and annotated to 10 metabolic pathways, and 21 DEGs of B vs. CK were enriched and annotated to 83 metabolic pathways. Based on the enrichment results $(Q$-value $<0.05)$ (Figure 5D), there were nine significantly enriched pathways in A vs. CK, mainly including protein digestion and absorption, olfactory transduction, inositol phosphate metabolism, starch and sucrose metabolism, and calcium signaling pathway. There were 21 significantly enriched pathways in B vs. CK, mainly including phosphonate and phosphinate metabolism, DNA replication, adherens junction, amyotrophic lateral sclerosis, peroxisome, and inositol phosphate metabolism. The inositol phosphate metabolism pathway was shared by the two comparative analysis groups.

\section{Transcription Factor (TF) Analyses}

Changes in the expression patterns of TFs can affect the expression levels of regulatory genes. A total of 30 TFs (20 downregulated, 10 upregulated) were identified among the DEGs of A vs. CK. They belonged to 11 TF families, mainly including zf-C2H2 (10), TF_others (6), ZBTB (3), bHLH (2), Homeobox (2), and MBD (2). A total of 39 TFs (24 downregulated, 15 upregulated) were identified among the DEGs of $\mathrm{B}$ vs. $\mathrm{CK}$. They belonged to $12 \mathrm{TF}$ families, mainly including zfC2H2 (10), Homeobox (5), bHLH (4), TF_others (4), MYB (3), TF_bZIP (3), ZBTB (3), and zf-LITAF-like (3). In the two comparative analysis groups, the number of downregulated 


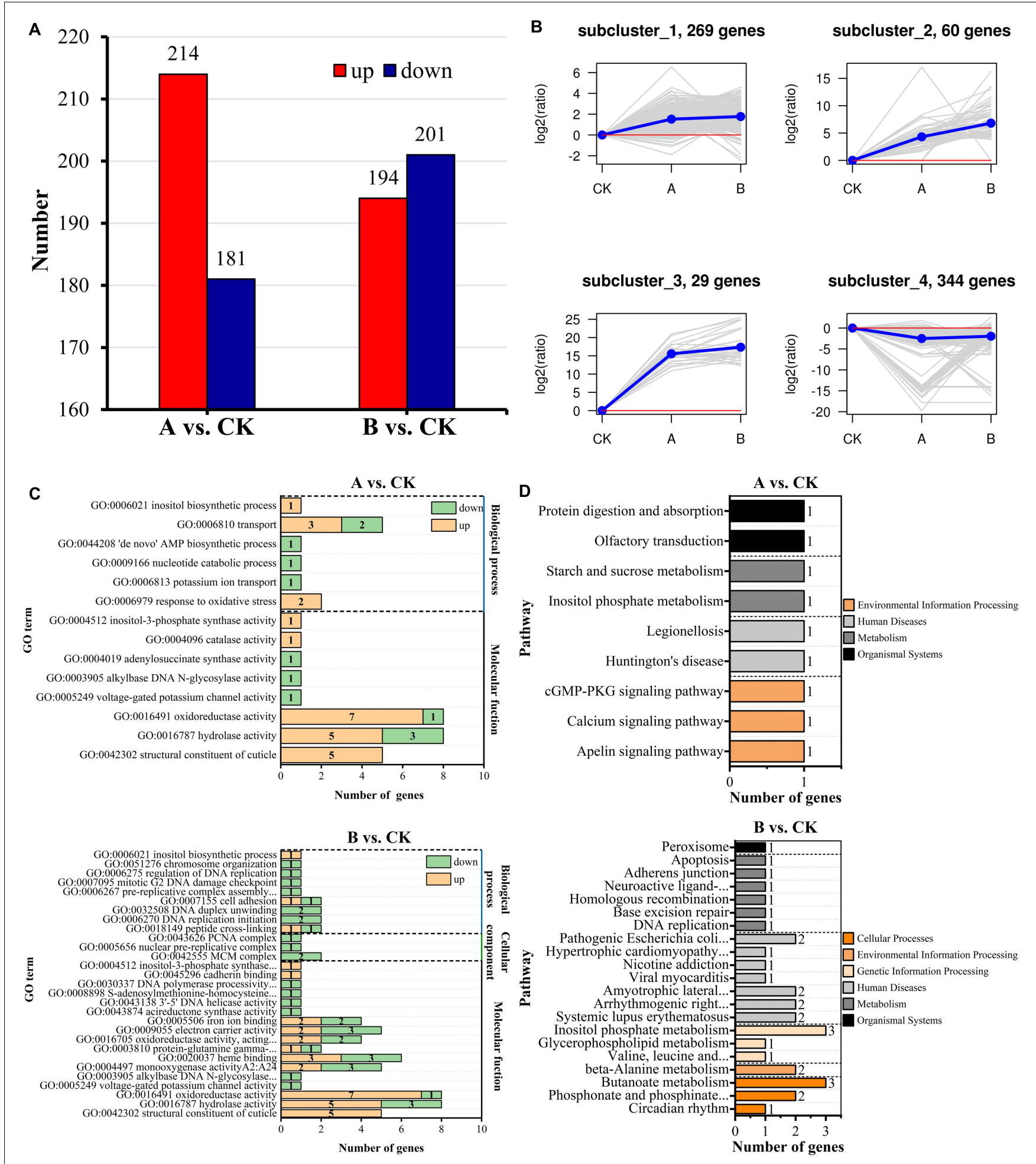

FIGURE 5 | Identification, clustering, and functional enrichment analyses of DEGs. (A) Statistics of the number of DEGs. (B) Analyses of expression patterns. (C) Significant enrichment map of GO terms. (D) Significant enrichment map of KEGG pathways.

TFs was greater than the number of upregulated TFs. The expression pattern of each TF is shown in Figure 6A and Supplementary Table 5. It is speculated that the expression of regulatory genes in A. glabripennis larvae is affected by the differential expression of TFs, and plays a role in the insect immune response to Bt toxin. 

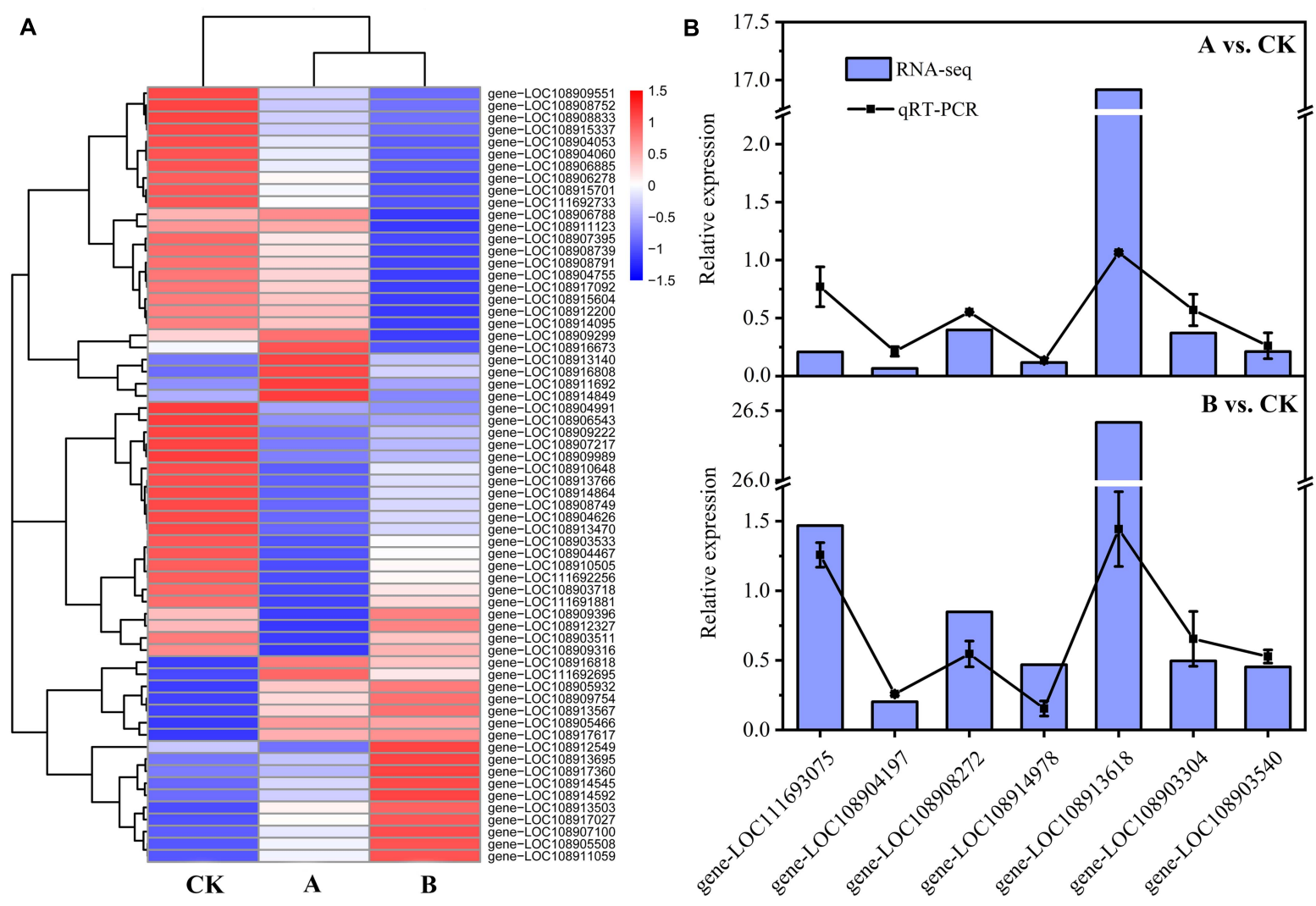

FIGURE 6 | Heat map of TF expression and RT-qPCR validation. (A) Heat map of TF expression. (B) RT-qPCR analyses to confirm expression patterns indicated by RNA-seq analyses. Genes were randomly selected for RT-qPCR. The RT-qPCR results are presented as the mean average of $2^{-\Delta \Delta C T}$ values. The RNA-seq results are presented as the Fold Change. gene-LOC111693075, sugar transporter ERD6-like 4; gene-LOC108904197, probable transaldolase; gene-LOC108908272, cathepsin B-like; gene-LOC108914978, uncharacterized LOC108914978; gene-LOC108913618, uncharacterized LOC108913618; gene-LOC108903304, glutathione S-transferase-like; gene-LOC108903540, endoglucanase.

\section{RT-qPCR Verification}

Seven genes were randomly selected for RT-qPCR analyses to verify the accuracy of the RNA-seq results. The results of comparison of the RT-qPCR results with the RNA-seq data are shown in Figure 6B. In the two comparative analysis groups, the RT-qPCR and RNA-seq results for six genes showed little difference, while the results for one gene (gene-LOC108913618) differed greatly. However, the expression patterns of the genes as determined by RT-qPCR and RNA-seq were the same, which demonstrated the reliability of the transcriptome data obtained in this study.

\section{Identification of Genes in A. glabripennis Larvae That Are Differentially Expressed in Response to Bt Toxin}

Based on the gene IDs of the DEGs, annotation information was retrieved from NCBI, and the DEGs related to Bt toxin mechanism, digestive enzymes, detoxification enzymes, and protective enzymes of $A$. glabripennis larvae were identified (Supplementary Table 6). Based on the expression level (FPKM) data, a heat map of the 59 identified DEGs was drawn (Figure 7), and the expression differences in genes related to the Bt toxin response between the two transgenic lines and CK were analyzed.

\section{Bt Prototoxin Activation- and Digestion-Related Proteases}

Trypsin and chymotrypsin are important digestive enzymes and play a critical role in the activation of $\mathrm{Bt}$ prototoxin. In this study, the expression of genes encoding prototoxin activationrelated enzymes changed in A. glabripennis larvae fed poplar feed containing Cry3A toxin. Four trypsin-related genes (two upregulated, two downregulated) and two chymotrypsin-related genes (both upregulated) were identified among the DEGs of A vs. CK (Figure 7 and Supplementary Table 6), but were not found in the DEGs of B vs. CK. If the conditions of DEG identification were set as $P<0.1$ and $\left|\log _{2} \mathrm{FC}\right|>1$, two trypsin-related genes (one upregulated, $\log _{2} \mathrm{FC}=1.237$ and one downregulated, $\log _{2} \mathrm{FC}=-1.128$ ) and two chymotrypsin-related genes (upregulated, $\log _{2} \mathrm{FC}=1.236$ and 1.056) were identified.

After food reaches the midgut, a variety of digestive enzymes participate in digestion and absorption. In addition to the two serine proteases, trypsin and chymotrypsin, mentioned above, other serine proteases, cathepsin, and carboxypeptidase, as well 


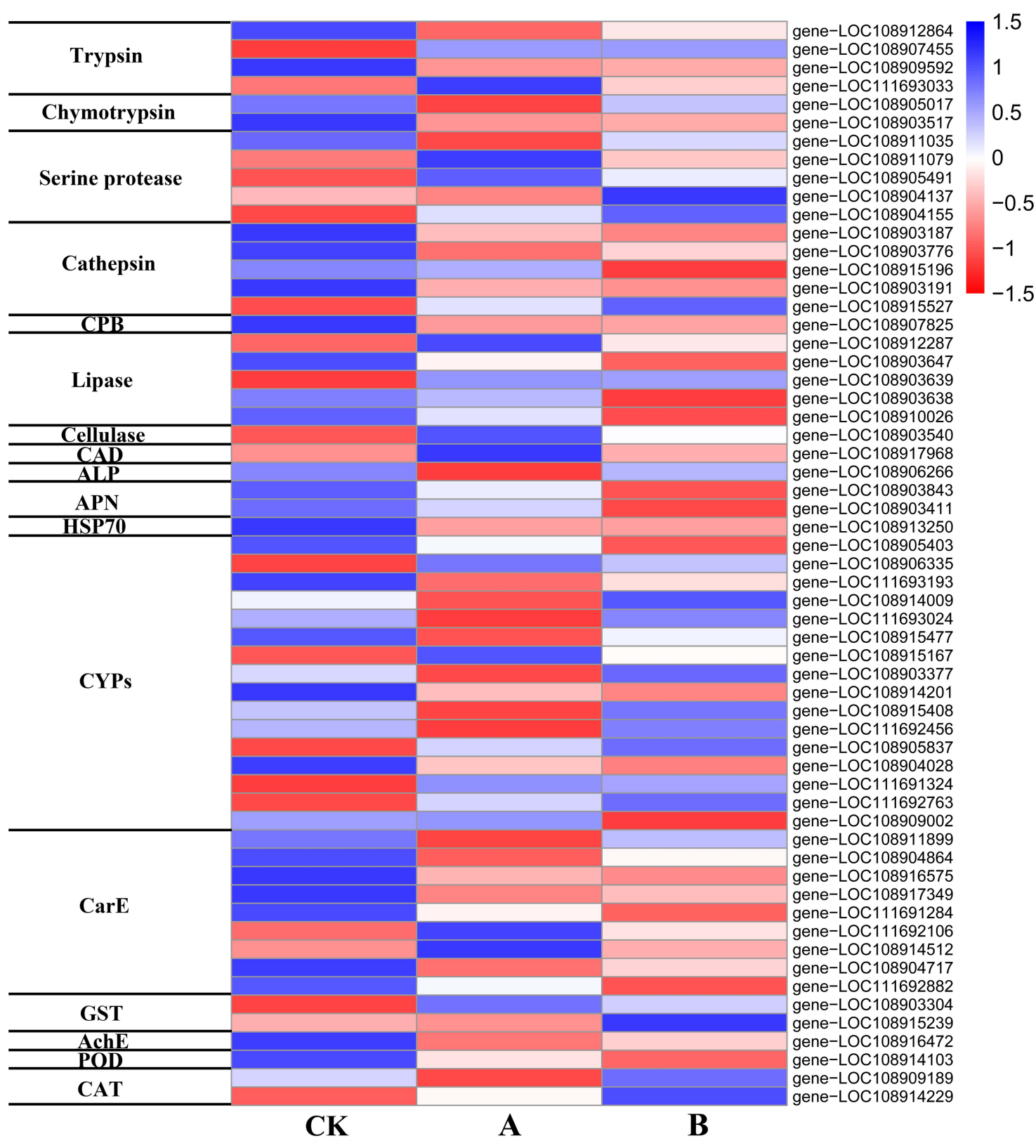

FIGURE 7 | Heat map of DEG expression.

as lipase, and cellulase are also involved. Three serine proteaserelated genes (one upregulated, two downregulated), three cathepsin-related genes (two cathepsin L and one cathepsin B, upregulated), three lipase-related genes (one upregulated and two downregulated), and one cellulase-related gene (endoglucanase, downregulated) (Figure 7 and Supplementary Table 6) were identified among DEGs of A vs. CK. Two serine proteaserelated genes (downregulated), four cathepsin-related genes (two cathepsin L and one cathepsin B, upregulated; one cathepsin L, downregulated), one carboxypeptidase $B$ gene (upregulated), and three lipase-related genes (two upregulated, one downregulated) were identified among the DEGs of B vs. CK (Figure 7 and
Supplementary Table 6). After A. glabripennis larvae were fed poplar feed containing Bt toxin, the expression of various digestive enzymes changed, which may play a role in the insect immune response to Bt toxin.

\section{Potential Bt Toxin-Binding Receptor Protein-Related DEGs}

After Bt toxin is hydrolyzed and toxic peptide molecules are released, they must bind with specific receptors to perform their function. After the A. glabripennis larvae were fed poplar feed containing Cry3A toxin, the expression of genes encoding potential Bt toxin-binding receptors changed significantly 
(Figure 7 and Supplementary Table 6). Three Bt toxin-binding receptor-related genes were identified among the DEGs of A vs. $\mathrm{CK}$, including one cadherin (CAD) gene (downregulated), one alkaline phosphatase (ALP) 4-like gene (upregulated), and one HSP70 gene (upregulated). Three toxin-binding receptor-related genes were identified among the DEGs of B vs. CK, including two aminopeptidase $\mathrm{N}$ (APN)-related genes (upregulated) and one HSP70 gene (upregulated).

\section{Detoxification Enzyme-Related DEGs}

Cytochrome P450 monooxygenase (CYP), carboxylesterase (CarE), and glutathione S-transferase (GST) are three detoxification enzymes that play key roles in resistance metabolism in insects. In addition, acetyl cholinesterase (AchE) is a detoxification enzyme that plays an important role in decomposing exogenous toxins and maintaining normal physiological metabolism in insects. In this study, the expression levels of detoxification enzyme-related genes changed significantly in A. glabripennis larvae fed poplar feed containing Cry3A toxin (Figure 7 and Supplementary Table 6). Eleven CYP-related genes (nine upregulated, two downregulated), seven CarE-related genes (five upregulated, two downregulated), one GST gene (downregulated), and one AchE gene (upregulated) were identified among the DEGs of A vs. CK. Seven CYP-related genes (three upregulated, four downregulated), five CarE-related genes (upregulated), one GST gene (downregulated), and one AchE gene (upregulated) were identified among the DEGs of $\mathrm{B}$ vs. CK. Most of the detoxification enzyme-related genes identified in this study were upregulated, indicating that the detoxification metabolic mechanism was activated after $A$. glabripennis larvae fed on plant materials containing Bt toxin.

\section{Protective Enzyme-Related DEGs}

The expression of protective enzymes in A. glabripennis larvae fed poplar feed containing Cry3A toxin also changed (Figure 7 and Supplementary Table 6). One peroxidase (POD)-related gene (upregulated) and one catalase (CAT)-related gene (upregulated) were identified among the DEGs of A vs. CK. One POD-related gene (upregulated) and one CAT-related gene (downregulated) were identified among the DEGs of B vs. CK.

\section{DISCUSSION}

\section{Exogenous Gene Expression and Effect on Insect Resistance in Dual Bt Toxin Populus x euramericana 'Neva' Transgenic Plants}

The use of combinations of multiple transgenes to improve plant traits is a new trend in transgenic plant research. Dong et al. (2014) and Zhou et al. (2020) introduced several exogenous genes in the same expression vector into poplar and obtained transgenic plants with multiple resistance traits. In this study, Cry $1 A c$ and Cry $3 A$ genes were simultaneously transformed into Populus $\times$ euramericana 'Neva' and 16 transgenic lines were obtained through kanamycin screening and confirmed by PCR. The dual Bt toxin genes were expressed at both the transcriptional and translational levels. The Cry3A toxin content was much higher than that of CrylAc toxin, similar to previous research results (Dong et al., 2014; Zhou et al., 2020). This difference may be related to gene structure and promoter type used. In this study, most of the transgenic lines had strong insecticidal effects against $H$. cunea and M. troglodyta L1 larvae, and P. versicolora L1 and L2 larvae and adults, but poor toxicity against A. glabripennis larvae, although their growth and development were significantly inhibited. The levels of resistance of different transgenic lines to the same target insect also differed, which may be related to transgene insertion position and copy number.

Our laboratory previously performed studies on transgenic Populus $\times$ euramericana 'Neva' expressing dual Bt and salttolerance multi-gene. The transgenic lines obtained exhibited high resistance to $P$. versicolora larvae, but poor resistance to H. cunea larvae (Yang et al., 2016; Liu et al., 2016). Compared with previous studies, the insecticidal activity of the transgenic lines against $H$. cunea L1 larvae was improved, and the corrected mortality rates of some lines reached $100 \%$, which may be related to adding an AtADH $5^{\prime}$-UTR enhancer upstream of the Cry1Ac gene. The AtADH $5^{\prime}$-UTR enhanced the expression of GUS by 150- and 87-fold in tobacco (Nicotiana tabacum) and Arabidopsis thaliana, respectively (Sugio et al., 2008). However, there were no significant differences in the insecticidal effects on $P$. versicolora L1 or L2 larvae. In this study, by contrast, the corrected mortality rates of $P$. versicolora adults fed transgenic lines reached as high as $100 \%$, while the corrected mortality rates of $P$. versicolora third instar larvae were only $81.71 \%$ of that observed by Liu et al. (2016). The improved insecticidal effects of Cry $3 \mathrm{~A}$ may be related to the type of promoter used or the order of genes in the T-DNA. CoYMV has shown even greater activity than CaMV35S in tobacco and maize suspension cells (Medberry et al., 1992). Qiu et al. (2017) found that in tobacco transformed by dual Bt genes, the sequence and orientation of Bt gene expression cassette in the T-DNA could affect the expression content of Bt gene.

In this study, the insecticidal effects of individual transgenic lines against Lepidopteran $H$. cunea and $M$. troglodyta larvae differed, which may be related to the sensitivity of the insects to Bt toxin. The sensitivity to Bt toxin differs among insect species, possibly due to their different enzyme systems (Wang et al., 2018). In this study, the corrected mortality rates of $P$. versicolora larvae and adults fed transgenic lines $7,9,11$, and 15 reached $100 \%$, while those of newly hatched A. glabripennis larvae were only $10 \%$, indicating that the leaf beetles were more sensitive to $\mathrm{Bt}$ toxin than longicorn beetles. Compared with $\mathrm{CK}$, the transgenic lines significantly inhibited the growth and development of A. glabripennis larvae. The midgut cellulase activities of the A. glabripennis larvae fed transgenic lines were lower than those fed $\mathrm{CK}$, and there was a significant correlation between the cellulase activity and the body weight of the larvae, indicating that Bt toxin affected the ability of the larvae to digest cellulose by inhibiting cellulase activity, thus inhibiting larval growth and development.

In this study, transgenic lines with high resistance to Lepidopteran and Coleopteran pests were produced. These lines provide new materials for developing transgenic Populus $\times$ euramericana 'Neva' plants expressing 
insect-resistance genes. Whether exogenous genes can be stably expressed in transgenic plants is an important factor affecting their application. The insect resistance of some transgenic poplar lines with high Bt toxin content has been observed to decrease with increasing tree age (Ren et al., 2018). The reason for this phenomenon may be that external environmental conditions and plant growth and development affect the expression and stability of exogenous genes through inactivation and silencing (Xia et al., 2000). At the same time, high Bt toxin gene expression may affect the growth and development of the transgenic plants (Ren et al., 2017). In future studies, the growth and development of dual Bt toxin Populus $\times$ euramericana 'Neva' transgenic plants and the stability of exogenous gene expression and insect resistance will be examined.

\section{Transcriptional Response of A. glabripennis Larvae to $\mathrm{Bt}$ Toxin}

The expression of various genes in A. glabripennis larvae changed significantly after feeding on poplar feed containing Bt toxin. These genes may be involved in the immune response of $A$. glabripennis larvae to Bt toxin.

The activation of $\mathrm{Bt}$ prototoxin is a complex process in which trypsin and chymotrypsin play an important role. The activities of trypsin and chymotrypsin were increased by feeding the fifth instar larvae of Helicoverpa armigera (Lepidoptera) with Cry1Ac prototoxin for $12 \mathrm{~h}$ (Wei et al., 2016). However, compared with the control, the expression of trypsin-like gene was downregulated in Tenebrio molitor (Coleoptera) fed with Cry3Aa for $24 \mathrm{~h}$ (Oppert et al., 2012). This may be related to species and Cry toxin types. In this study, two trypsin and two chymotrypsin-related genes were upregulated and two trypsin genes were downregulated in A vs. CK, similar to the results of Yang et al. (2018) who examined the transcriptional response of Cnaphalocrocis medinalis (Lepidoptera) to Cry1C.

Serine protease is an important protease in the digestive and innate immune systems of insects, and changes in its expression may be related to insect resistance to Bt toxin (Yang et al., 2018). In this study, the differential expression of serine protease may be involved in the activation or degradation of Bt toxin. Studies have shown that cysteine and aspartic proteases are the main digestive enzymes of Coleoptera (Wu et al., 2016). After the third instar larvae of Monochamus alternatus (Coleoptera) were treated orally with Bt toxin insecticides, expression of the cathepsin L (cysteine protease) gene in the midgut was downregulated. It is speculated that Bt toxin may be toxic to $M$. alternatus by disrupting its development (Lin et al., 2016). After ingestion of eCry3.1Ab, the expression of a cathepsin B-like (cysteine protease) gene in susceptible strains of western corn rootworm was significantly increased (Zhao et al., 2019). In this study, cathepsin L and cathepsin B were differentially expressed in A. glabripennis larvae that fed on dual Bt toxin Populus $\times$ euramericana 'Neva' transgenic plants and they were mainly upregulated. Differences between this study and others may be related to the different species examined or the complex regulatory mechanism of Bt toxin. Cellulase is an important digestive enzyme that allows A. glabripennis to digest cellulose and obtain nutrients, and plays an important role in A. glabripennis growth and development. gene-LOC108903540, annotated as endoglucanase gene, performs the functions of carbohydrate metabolism process (go:0005975) and cellulase activity (go:0008810). In this study, the endoglucanase gene was differentially expressed in A vs CK, but not in B vs CK due to $P>0.05$. However, the expression of endoglucanase gene decreased in both of them, and the cellulase activity in the midgut of longicorn larvae was significantly lower than that in the control. The growth and development of larvae in $\mathrm{A}$ and $\mathrm{B}$ were also significantly inhibited, indicating that Bt toxin could affect the growth and development of A. glabripennis larvae by inhibiting the cellulase activity.

At present, there are no reports of CAD, ALP, or APN acting as $\mathrm{Bt}$ toxin-binding receptors in A. glabripennis larvae. This study provides valuable information for further studies to determine whether these proteins are Bt toxin receptors in A. glabripennis larvae. Contreras et al. (2013) found a type of cadherinlike Cry3Aa receptor in Tribolium Castanea (Coleoptera: Tenebrionidae) and confirmed through gene silencing that the cadherin-like protein was a representative Cry receptor. In this study, one $C A D$ gene was downregulated in $\mathrm{A}$ vs. CK, which was similar to the response of western corn root worm to Cry3Bb1 (Rault et al., 2018), which may be related to the pore formation model. Both APN and ALP are glycosylphosphatidylinositol (GPI)-anchored proteins, which play a role in the process of membrane insertion and pore formation. Many studies have shown that mutations of APN1 or downregulated expression of $A P N 1$ and $A L P 2$ are associated with $\mathrm{Bt}$ toxin resistance (Zhang et al., 2009; Tiewsiri and Wang, 2011), but no studies have reported that the upregulated expression of APN1 or $A L P 2$ is associated with $\mathrm{Bt}$ toxin resistance. In this study, APN- and ALP-related genes were upregulated, which may show their own enzyme activities and play a role in their respective metabolism.

CYPs, CarE, and GST are important enzymes that play a central role in detoxification and metabolism of exogenous substances in insect systems. Several genes of the CYP6 family are related to resistance in insects, and CYP6 gene expression is upregulated as the main mechanism of resistance (Kim et al., 2017; Lee et al., 2018). In this study, CYP6, CYP4, CYP 12, and other CYP family genes were identified, and most of them were upregulated. They perform multiple functions such as oxidoreductase activity, metal ion binding, and binding (Supplementary Table 6), similar to the results of Behrens et al. (2014) in Tribolium castaneum. The CarE detoxification enzyme is involved in various functions including digestion and detoxification in A. longicornis (Coleoptera) (Han et al., 2019). In this study, most CarE-related genes were upregulated, and may play a certain role in detoxification during the immune response to Bt toxin. Glutathione S-transferases (GSTs) are a class of multifunctional detoxification enzymes, which are involved in the metabolism of many endogenous and exogenous toxic substances and the formation of drug resistance (Li et al., 2007). Studies have shown that a high level of GST expression is related to the insecticide resistance mechanism of insects (Bass and Field, 2011). In addition, GSTs can also protect cells from oxidative damage and intercellular transport of hormones, endogenous 
metabolites and exogenous compounds (Feng et al., 1999). In this study, gene-LOC108903304, annotated as glutathione S-transferase-like gene, was downregulated in A vs CK and B vs $\mathrm{CK}$, indicating that $\mathrm{Bt}$ toxin may affect the detoxification and intercellular transport of toxin and larval resistance by affecting the expression of GST. The upregulated expression of the most detoxification enzymes indicates the induction of a metabolic detoxification mechanism in A. glabripennis larvae after feeding on poplar feed containing Bt toxin to reduce its toxicity.

When insects feed on Bt toxin, the balance of protective enzymes may be disrupted, potentially leading to excessive and damaging levels of $\mathrm{O}^{2-}$. Insects can protect and maintain normal functions by increasing the activities of protective enzymes, thus producing resistance to Bt toxin (Zhang Z. et al., 2011). Yang et al. (2018) found that POD gene expression patterns were both upregulated and downregulated, while CAT gene expression was downregulated in the transcriptional response of C. medinalis to Cry1C. In this study, POD gene expression was upregulated, while changes in CAT gene expression were not consistent between the two groups. Many studies have shown that increased POD expression is an emergency response to Bt toxin (Guo et al., 2011; Zhang Z. et al., 2011).

In conclusion, this study obtained dual Bt toxin Populus $\times$ euramericana 'Neva' transgenic lines with high insect resistance, which provides new materials for developing insect-resistant poplars. A. glabripennis larvae responded to $\mathrm{Bt}$ toxin by regulating the expression of genes related to $\mathrm{Bt}$ prototoxin activation, digestive enzymes, binding receptors, detoxification enzymes, and protective enzymes, which lays a theoretical foundation for establishing resistance to A. glabripennis in poplar, and provides a basis for exploring the mechanism of $\mathrm{Bt}$ toxin against Cerambycidae insects.

\section{DATA AVAILABILITY STATEMENT}

The original contributions presented in the study are publicly available. This data can be found here: The A. glabripennis larvae transcriptome data have been uploaded to Sequence Read Archive (https://www.ncbi.nlm.nih.gov/sra) under Bioproject PRJNA691113.

\section{REFERENCES}

Bass, C., and Field, L. M. (2011). Gene amplification and insecticide resistance. Pest Manag. Sci. 67, 886-890. doi: 10.1002/Ps.2189

Behrens, S., Peu, R., Milutinovic, B., Eggert, H., Esser, D., Rosenstiel, P., et al. (2014). Infection routes matter in population-specific responses of the red flour beetle to the entomopathogen Bacillus thuringiensis. BMC Genomics 15:445. doi: 10.1186/1471-2164-15-445

Bravo, A., Gill, S. S., and Soberón, M. (2007). Mode of action of Bacillus thuringiensis Cry and Cyt toxins and their potential for insect control. Toxicon 49, 423-435. doi: 10.1016/j.toxicon.2006.11.022

Contreras, E., Schoppmeier, M., Real, M. D., and Rausell, C. (2013). Sodium solute symporter and cadherin proteins act as Bacillus thuringiensis Cry3Ba toxin

\section{AUTHOR CONTRIBUTIONS}

YR conducted the experiments, analyzed the data, and wrote the manuscript. XZ conducted the experiments and analyzed the data. YD designed the experiments and edited the manuscript. JZ edited the manuscript. JW and MY designed the experiments and edited the manuscript. All authors contributed to the article and approved the submitted version.

\section{FUNDING}

This work was supported by the National Key Program on Transgenic Research (2018ZX08020002) and the Basic Research Plan Project of Hebei Province (18966801D).

\section{ACKNOWLEDGMENTS}

We would like to thank Professor Min Chen of Beijing Forestry University for providing the eggs of $H$. cunea and to Textcheck (https://www.textcheck.com) for English language editing of this manuscript.

\section{SUPPLEMENTARY MATERIAL}

The Supplementary Material for this article can be found online at: https://www.frontiersin.org/articles/10.3389/fpls.2021. 660226/full\#supplementary-material

Supplementary Figure 1 | Comparison of insect experiments. (A) H. cunea L1 larvae. (B) P. versicolora L1 larvae. (C) P. versicolora L2 larvae. (D) P. versicolora adults. CK, non-transgenic Populus $\times$ euramericana 'Neva'; T, transgenic line.

Supplementary Table 1 | Information of primers used in the experiment.

Supplementary Table 2 | Correlation coefficient.

Supplementary Table 3 | Sequencing data evaluation statistics.

Supplementary Table 4 | The shared DEGs were identified in two comparative analysis groups.

Supplementary Table 5 | The expression information of TFs.

Supplementary Table 6 | DEGs identified in the transcriptome of $A$. glabripennis larvae in response to Bt toxin.

functional receptors in Tribolium castaneum. J. Biol. Chem. 288, 18013-18021. doi: 10.1074/Jbc.M113.474445

Ding, L., Chen, Y., Wei, X., Ni, M., Zhang, J., Wang, H., et al. (2017). Laboratory evaluation of transgenic Populus davidiana $\times$ Populus bolleana expressing Cry1Ac + SCK, Cry1Ah3, and Cry9Aa3 genes against gypsy moth and fall webworm. PLoS One 12:e0178754. doi: 10.1371/journal.pone.0178754

Dong, Y., Du, S. S., Zhang, J., Yang, M. S., and Wang, J. M. (2014). Differential expression of dual Bt genes in transgene poplar Juba (Populus deltoides cv. 'Juba') transformed by two different transformation vectors. Can. J. For. Res. 45, 60-67. doi: 10.1139/cjfr-2014-0335

Feng, Q. L., Davey, K. G., Pang, A. S. D., Primavera, M., Ladd, T. R., Zheng, S. C., et al. (1999). Glutathione S-transferase from the spruce budworm, Choristoneura fumiferana: identification, characterization, localization, cDNA 
cloning, and expression. Insect Biochem. Molec. 29, 779-793. doi: 10.1016/ S0965-1748(99)00048-X

Flores-Escobar, B., Rodríguez-Magadan, H., Bravo, A., Soberón, M., and Gómez, I. (2013). Differential role of Manduca sexta aminopeptidase-N and alkaline phosphatase in the mode of action of CrylAa, CrylAb, and CrylAc toxins from Bacillus thuringiensis. Appl. Environ. Microbiol. 79:4543. doi: 10.1128/AEM. 01062-13

Fritz, M. L., Nunziata, S. O., Guo, R., Tabashnik, B. E., and Carrière, Y. (2020). Mutations in a novel cadherin gene associated with Bt resistance in Helicoverpa zea. G3 (Bethesda) 10, 1563-1574. doi: 10.1534/g3.120.401053

Guo, J. Y., Wu, G., and Wan, F. H. (2011). Temporal allocation of metabolic tolerance to transgenic Bt cotton in beet armyworm, Spodoptera exigua (Hübner). Sci. China Life Sci. 54, 152-158. doi: 10.1007/s11427-010-4133-y

Han, X., Lu, C., Hua, Y., Lin, H., Shi, Y., Wu, S., et al. (2019). Phylogenetic analysis of transcriptome and three detoxification enzyme families related genes in Anoplophora chinensis (Coleoptera: Cerambycidae). Sci. Silv. Sin. 55, 104-113. doi: 10.11707/j.1001-7488.20190512

Jin, M., Liao, C., Chakrabarty, S., Wu, K., and Xiao, Y. (2019). Comparative proteomics of peritrophic matrix provides an insight into its role in CrylAc resistance of cotton bollworm Helicoverpa armigera. Toxins 11:92. doi: 10.3390/ toxins 11020092

Kim, H. S., Han, J., Kim, H. J., Hagiwara, A., and Lee, J. S. (2017). Identification of 28 cytochrome $\mathrm{P} 450$ genes from the transcriptome of the marine rotifer Brachionus plicatilis and analysis of their expression. Comp. Biochem. Physiol. Part D Genomics Proteomics 23, 1-7. doi: 10.1016/j.cbd.2017.04.005

Lee, B. Y., Kim, D. H., Kim, H. S., Kim, B. M., Han, J., and Lee, J. S. (2018). Identification of 74 cytochrome $\mathrm{P} 450$ genes and co-localized cytochrome $\mathrm{P} 450$ genes of the CYP2K, CYP5A, and CYP46A subfamilies in the mangrove killifish Kryptolebias marmoratus. BMC Genomics 19:7-18. doi: 10.1186/s12864-0174410-2

Li, X., Luo, Y., Yan, X., Tian, G., Nian, Y., and Sun, H. (2011). Cellulase in larvae of Asian longhorn beetle Anoplophora glabripennis. Sci. Silv. Sin. 47, 204-207. doi: 10.11707/j.1001-7488.20111133

Li, X., Schuler, M. A., and Berenbaum, M. R. (2007). Molecular mechanisms of metabolic resistance to synthetic and natural xenobiotics. Annu. Rev. Entomol. 52, 231-253. doi: 10.1146/annurev.ento.51.110104.151104

Lin, T., Cai, Z., Wu, H., and Luo, L. (2016). Changes in midgut gene expression following Bacillus thuringiensis (Bacillales: Bacillaceae) infection in Monochamus alternatus (Coleoptera: Cerambycidae). Fla. Entomol. 99, 60-66. doi: 10.1653/024.099.0111

Liu, D., Zhang, J., Dong, Y., Zhang, X., Yang, M., and Gao, B. (2016). Genetic transformation and expression of Cry1Ac-Cry3A-NTHK1 genes in populus $\times$ euramericana "Neva". Acta. Physiol. Plant 38:177. doi: 10.1007/s11738-0162195-6

Lu, H., Wu, W., Chen, Y., Zhang, X., Devare, M., and Thies, J. E. (2010). Decomposition of $B t$ transgenic rice residues and response of soil microbial community in rapeseed-rice cropping system. Plant Soil 336, 279-290. doi: 10.1007/s11104-010-0476-1

Medberry, S. L., Lockhart, B. E., and Olszewski, N. E. (1992). The Commelina yellow mottle virus promoter is a strong promoter in vascular and reproductive tissues. Plant Cell 4, 185-192. doi: 10.1105/tpc.4.2.185

Mi, X., Ji, X., Yang, J., Liang, L., Si, H., Wu, J., et al. (2015). Transgenic potato plants expressing cry $3 A$ gene confer resistance to Colorado potato beetle. C. $R$. Biol. 338, 443-450. doi: 10.1016/j.crvi.2015.04.005

Niu, X., Kassa, A., Hasler, J., Griffin, S., Perez-Ortega, C., Procyk, L., et al. (2020). Functional validation of DvABCB1 as a receptor of Cry3 toxins in western corn rootworm, Diabrotica virgifera virgifera. Sci. Rep. 10:15830. doi: 10.1038/ s41598-020-72572-9

Niu, X. Y., Huang, D. Z., Yang, M. S., Wei, X. P., Li, X. F., and Fu, X. S. (2011). Expression of toxic protein in six transgenic poplar strains with BtCry3A gene and identification of their resistance against Apriona germari. Sci. Seric. 37, 593-599. doi: 10.3969/j.issn.0257-4799.2011.04.001

Oppert, B., Dowd, S. E., Bouffard, P., Li, L., Conesa, A., Lorenzen, M. D., et al. (2012). Transcriptome profiling of the intoxication response of Tenebrio molitor larvae to Bacillus thuringiensis Cry3Aa protoxin. PLoS One 7:e34624. doi: 10. 1371/journal.pone.0034624

Prabu, S., Jing, D., Shabbir, M. Z., Yuan, W., Wang, Z., and He, K. (2020). Contribution of phenoloxidase activation mechanism to Bt insecticidal protein resistance in Asian corn borer. Int. J. Biol. Macromol. 153, 88-99. doi: 10.1016/ j.ijbiomac.2020.03.003

Qiu, T., Dong, Y., Ren, Y., Wang, J., Yang, M., and Zhang, J. (2017). Effects of the sequence and orientation of an expression cassette in tobacco transformed by dual Bt genes. Plasmid 89, 1-8. doi: 10.1016/j.plasmid.2016.11.003

Rault, L. C., Siegfried, B. D., Gassmann, A. J., Wang, H., Brewer, G. J., and Miller, N. J. (2018). Investigation of Cry3Bb1 resistance and intoxication in western corn rootworm by RNA sequencing. J. Appl. Entomol. 142, 921-936. doi: $10.1111 /$ jen. 12502

Ren, Y., Zhang, J., Wang, G., Liu, X., Li, L., Wang, J., et al. (2018). The relationship between insect resistance and tree age of transgenic triploid Populus tomentosa plants. Front. Plant Sci. 9:53. doi: 10.3389/fpls.2018.00053

Ren, Y., Zhang, W., Huang, Y., Zhang, Y., Wang, J., and Yang, M. (2020). Transcriptome analysis reveals that Populus tomentosa hybrid poplar 741 responds to blue light treatment by regulating growth-related genes and their metabolic pathways. Ind. Crops Prod. 152:112512. doi: 10.1016/j.indcrop.2020. 112512

Ren, Y. C., Dong, Y., Zhang, W. L., and Yang, M. S. (2017). Superposition effect of CrylAc gene in transgenic Bt tobacco (Nicotiana tabacum). J. Agric. Biotechnol. 25, 1903-1917. doi: 10.3969/j.issn.1674-7968.2017.12.001

Ruiz-Arroyo, V. M., García-Robles, I., Ochoa-Campuzano, C., Goig, G. A., Zaitseva, E., Baaken, G., et al. (2017). Validation of ADAM10 metalloprotease as a Bacillus thuringiensis Cry3Aa toxin functional receptor in Colorado potato beetle (Leptinotarsa decemlineata). Insect Mol. Biol. 26, 204-214. doi: 10.1111/ imb. 12285

Shabbir, M. Z., Zhang, T. T., Wang, Z. Y., and He, K. L. (2019). Transcriptome and proteome alternation with resistance to Bacillus thuringiensis CrylAh toxin in Ostrinia furnacalis. Front. Physiol. 10:27. doi: 10.3389/fphys.2019.00027

Shi, W., Ding, S. Y., and Yuan, J. S. (2011). Comparison of insect gut cellulase and xylanase activity across different insect species with distinct food sources. Bioenerg. Res. 4, 1-10. doi: 10.1007/s12155-010-9096-0

Sinegani, A. A. S., and Emtiazi, G. (2006). The relative effects of some elements on the DNS method in cellulase assay. J. Appl. Sci. Environ. Manage. 10, 93-96. doi: 10.4314/jasem.v10i3.17326

Sugio, T., Satoh, J., Matsuura, H., Shinmyo, A., and Kato, K. (2008). The 5'untranslated region of the Oryza sativa alcohol dehydrogenase gene functions as a translational enhancer in monocotyledonous plant cells. J. Biosci. Bioeng. 105, 300-302. doi: 10.1263/jbb.105.300

Tiewsiri, K., and Wang, P. (2011). Differential alteration of two aminopeptidases $\mathrm{N}$ associated with resistance to Bacillus thuringiensis toxin Cry1Ac in cabbage looper. Proc. Natl. Acad. Sci. U.S.A. 108, 14037-14042. doi: 10.1073/pnas. 1102555108

Wang, G., Dong, Y., Liu, X., Yao, G., Yu, X., and Yang, M. (2018). The current status and development of insect-resistant genetically engineered poplar in China. Front. Plant Sci. 9:1408. doi: 10.3389/fpls.2018.01408

Wang, G. L., and Fang, H. J. (2002). Plant genetic engineering. Beijing: Science press, 638-659.

Wang, G. Y., Yang, M. S., Huo, X. M., Wang, Y. P., and Li, S. S. (2012). Transformation of 741 poplar with double $B t$ genes and the insect-resistance of the transgenic plant. Sci. Silv. Sin. 48, 42-49. doi: 10.11707/j.1001-7488. 20120907

Wang, L., Wang, J., Ma, Y., Wan, P., Liu, K., Cong, S., et al. (2019). Transposon insertion causes cadherin mis-splicing and confers resistance to Bt cotton in pink bollworm from China. Sci. Rep. 9:7479. doi: 10.1038/s41598-019-43889-x

Wei, J., Liang, G., Wang, B., Zhong, F., Chen, L., Khaing, M. M., et al. (2016). Activation of Bt protoxin CrylAc in resistant and susceptible cotton bollworm. PLoS One 11:e0156560. doi: 10.1371/journal.pone.0156560

Wu, S., Zhu, X., Liu, Z., Shao, E., Rebeca, C. L., Guo, Y., et al. (2016). Identification of genes relevant to pesticides and biology from global transcriptome data of Monochamus alternatus Hope (Coleoptera: Cerambycidae) larvae. PLoS One 11:e0147855. doi: 10.1371/journal.pone.0147855

Xia, L., Wang, Y., and Guo, S. (2000). The stability of the expression of foreign genes in transgenic plants. Biotechnol. Bull. 3, 8-12. doi: 10.13560/j.cnki. biotech.bull.1985.2000.03.002

$\mathrm{Xu}, \mathrm{C}$., Wei, H., Wang, L., Yin, T., and Zhuge, Q. (2019). Optimization of the $\operatorname{cry} 1 A h 1$ sequence enhances the hyper-resistance of transgenic poplars to Hyphantria cunea. Front. Plant Sci. 10:335. doi: 10.3389/fpls.2019.00335 
Xu, L., Ferry, N., Wang, Z. Y., Zhang, J., Edwards, M. G., Gatehouse, A. M. R., et al. (2013). A proteomic approach to study the mechanism of tolerance to Bt toxins in Ostrinia furnacalis larvae selected for resistance to Cry1 Ab. Transgenic Res. 22, 1155-1166. doi: 10.1007/s11248-013-9718-3

Xu, S., Zhang, Y., Li, S., Chang, L., Wu, Y., and Zhang, J. (2020). Plastidexpressed Bacillus thuringiensis (Bt) $c r y 3 B b$ confers high mortality to a leaf eating beetle in poplar. Plant Cell Rep. 39, 317-323. doi: 10.1007/s00299-019-02492-0

Yang, R. L., Wang, A. X., Zhang, J., Dong, Y., Yang, M. S., and Wang, J. M. (2016). Genetic transformation and expression of transgenic lines of Populus $\mathrm{x}$ euramericana with insect-resistance and salt-tolerance genes. Genet. Mol. Res. 15:gmr8635. doi: 10.4238/gmr.15028635

Yang, Y., Xu, H., Lu, Y., Wang, C., and Lu, Z. (2018). Midgut transcriptomal response of the rice leaffolder, Cnaphalocrocis medinalis (Guenée) to CrylC toxin. PLoS One 13:e0191686. doi: 10.1371/journal.pone.0191686

Zhang, B., Chen, M., Zhang, X., Luan, H., Diao, S., Tian, Y., et al. (2011). Laboratory and field evaluation of the transgenic Populus alba $\times$ Populus glandulosa expressing double coleopteran-resistance genes. Tree Physiol. 31, 567-573. doi: 10.1093/treephys/tpr032

Zhang, C., Wang, J. M., Zhao, J., Pang, D. W., Zhang, D. J., and Yang, M. S. (2019). Expression characteristics of Bt gene in transgenic poplar transformed by different multi-gene vectors. Sci. Silv. Sin. 55, 61-70. doi: 10.11707/j.10017488.20190907

Zhang, S., Cheng, H., Gao, Y., Wang, G., Liang, G., and Wu, K. (2009). Mutation of an aminopeptidase $\mathrm{N}$ gene is associated with Helicoverpa armigera resistance to Bacillus thuringiensis Cry1 Ac toxin. Insect Biochem. Mol. Biol. 39, 421-429. doi: 10.1016/j.ibmb.2009.04.003
Zhang, X., Candas, M., Griko, N. B., Taussig, R., and Bulla, L. A. (2006). A mechanism of cell death involving an adenylyl cyclase/PKA signaling pathway is induced by the Cryl Ab toxin of Bacillus thuringiensis. Proc. Natl. Acad. Sci. U.S.A. 103, 9897-9902. doi: 10.1073/pnas.0604017103

Zhang, Z., Sun, J., and Li, Y. (2011). Effects of CrylAb protein from transgenic $\mathrm{Bt}$ rice on the activities of three protective enzymes in larvae of the leaf roller Cnaphalocis medinalis. Biotechnol. Bull 148-152. doi: 10.13560/j.cnki.biotech. bull.1985.2011.01.023

Zhao, Z., Meihls, L. N., Hibbard, B. E., Ji, T., Elsik, C. G., and Shelby, K. S. (2019). Differential gene expression in response to eCry3.1Ab ingestion in an unselected and eCry3.1Ab-selected western corn rootworm (Diabrotica virgifera virgifera LeConte) population. Sci. Rep. 9:4896. doi: 10.1038/s41598019-41067-7

Zhou, X., Dong, Y., Zhang, Q., Xiao, D., Yang, M., and Wang, J. (2020). Expression of multiple exogenous insect resistance and salt tolerance genes in Populus nigra L. Front. Plant Sci. 11:1123. doi: 10.3389/fpls.2020.01123

Conflict of Interest: The authors declare that the research was conducted in the absence of any commercial or financial relationships that could be construed as a potential conflict of interest.

Copyright () 2021 Ren, Zhou, Dong, Zhang, Wang and Yang. This is an open-access article distributed under the terms of the Creative Commons Attribution License (CC BY). The use, distribution or reproduction in other forums is permitted, provided the original author(s) and the copyright owner(s) are credited and that the original publication in this journal is cited, in accordance with accepted academic practice. No use, distribution or reproduction is permitted which does not comply with these terms. 